\section{Detection of glaucomatous optic nerve head by using Heidelberg topographic maps}

\begin{abstract}
Purpose To evaluate the capacity of a new topographic map analysis to detect abnormal optic discs from healthy ones in a new cohort of subjects.

Patients and methods Only one eye was chosen randomly from each subject included in the study. In total, 20 normal eyes with a normal visual field, an IOP of $<22 \mathrm{mmHg}$ and no history of glaucoma in their family, and 20 glaucomatous eyes with an abnormal visual field and an open angle were selected. All the subjects were examined with the Heidelberg Retina Tomograph (HRT, Heidelberg Engineering GMBH, Heidelberg, Germany) and Humphrey Perimeter, program 30-2 (Humphrey Instrument, Inc., San Leandro, CA, USA). Topographic maps analysis was performed to each HRT optic nerve head image. Sensitivity, specificity, and diagnostic precision were calculated.

Results When the topographic map analysis was applied to the group, a sensitivity of $80 \%$, a specificity of $75 \%$, and a diagnostic precision of $77.5 \%$ were obtained.

Conclusion Using the topographic map analysis, the HRT capacity to differentiate normal optic discs from those with glaucoma was similar to those already published in the literature, but using this technique there is no input from the observer to draw the contourline and reference plane is not necessary.
\end{abstract}

Eye (2007) 21, 609-613. doi:10.1038/sj.eye.6702285; published online 17 February 2006

Keywords: glaucoma; optic nerve head; contourline; reference plane; scanning laser ophthalmoscope; topographic map
M lester ${ }^{1,2}$, M Zanini ${ }^{3}$, P Vittone ${ }^{2}$ and G Calabria ${ }^{1}$

\section{Introduction}

It has been shown that Heidelberg Retina Tomograph (HRT, Heidelberg Engineering GMBH, Heidelberg, Germany) has a good sensitivity and specificity to detect glaucomatous optic nerve head $(\mathrm{ONH})$ changes. ${ }^{1,2}$ The observer can use different mathematical approaches to distinguish normal ONHs from abnormal ones. ${ }^{3-8}$ The Moordfield Regression Analysis or the linear discriminant function analysis are able to detect glaucomatous ONHs with a sensitivity and specificity around $80-90 \% .^{3-8}$ However, there are two weak points in the HRT methodology: the reference plane and the contourline. ${ }^{9}$

The observer has to draw the contourline on the outer edge of the rim and then the system is able to find a reference plane, which is fundamental to calculate some of the HRT parameters, such as rim area or volume, cup area, or volume, etc.

In 1999, Iester et al..$^{10}$ introduced a topographic map analysis able to analyse the $\mathrm{ONH}$ without drawing the contourline.

The purpose of this study was to evaluate the capacity of this topographic map analysis to detect glaucomatous optic discs from healthy ones by using a cohort of patient different from the one used to create the algorithm.

\section{Patients and methods}

This is a cross-sectional cohort study. In total, 40 subjects were included in this study and only one eye for each subject was randomly included in the study. The research followed the tenets of the Declaration of Helsinki and informed consent was obtained from all enrolled patients.

A total of 20 patients were randomly recruited from the patients of the glaucoma center of the University of Genoa and
${ }^{1}$ Clinica Oculistica, Department of Neurological Sciences, Ophthalmology, Genetic, University of Genoa, Genoa, Italy

${ }^{2}$ Division of Ophthalmology, G Gaslini Institute,

Genoa, Italy

${ }^{3}$ Department of Physics, University of Genoa, Genoa, Italy

\section{Correspondence:}

M lester,

University Eye Clinic, c/o V.le Teano 71/1, 16147 Genoa, Italy Tel: + 0103538455 ; Fax: + 0103538494 E-mail: iester@unige.it

Received: 23 October 2005 Accepted in revised form: 23 December 2005 Published online: 17 February 2006 
20 age-matched normal subjects were recruited from volunteers. Subjects were not excluded on the basis of age, gender, and media opacity. Subjects with a refractive error more than -7 diopters $(\mathrm{dB})$ were excluded and best-corrected visual acuity was $20 / 30$ or better.

Glaucomatous patients had to have an abnormal visual field as defined below and an open angle as judged by gonioscopy. Although it may have played a part in the initial referral to the clinic, the visual appearance of the $\mathrm{ONH}$ was not used as a criterion by which to exclude or include normal subjects or patients. ${ }^{11}$ Normal subjects had to have a normal visual field, an IOP of $<21 \mathrm{mmHg}$ on at least two separate occasions and no history of glaucoma in their family. ${ }^{11}$

All the include patients performed a visual field test and an HRT analysis.

\section{Visual field analysis}

All the subjects had a visual field examination by the Humphrey Field Analyzer, program 30-2, full threshold (HFA; Humphrey Instrument, Inc., San Leandro, CA, USA). A visual field was classified as glaucomatous if it had: (a) three adjacent points down by $5 \mathrm{~dB}$ with one of the points being down by at least $10 \mathrm{~dB}$; (b) two adjacent points down by $10 \mathrm{~dB}$; or (c) a $10 \mathrm{~dB}$ difference across the nasal horizontal meridian in at least two adjacent points. None of the points could be edge points except immediately above or below the nasal horizontal meridian. ${ }^{12}$ Only reliable fields were used, as determined by the reliability parameters: there had to be less than three fixation losses, and false-positive and false-negative responses both had to be $<10 \%$.

Mean deviation (MD) and corrected pattern standard deviation (CPSD) were calculated.

\section{Optic nerve head topographic analysis}

Optic disc images were analysed using the HRT, version 2.01S. The accuracy and reproducibility of this instrument have previously been described in detail. ${ }^{13-22}$ The HRT uses a scanning confocal laser imaging system, which produces a series of 32 confocal images of the back of the eye at consecutive focal planes, each $256 \times 256$ pixels. This series is converted by further processing into a single 3-D topographic image. The field of each image was $10^{\circ}$. Three images were obtained for each eye, and a single mean topographic image was further calculated after alignment and averaging.

The optic disc margin outline, at the inner edge of the scleral ring (Elshnig's ring), under visual guidance was drawn using a computer mouse system by a trained observer (MI). Then the program (Stereometric Measurements) calculated a number of predefined shape parameters. To determine some of these HRT parameters such as rim area and volume, cup area and volume, cup/ disc area ratio, mean retinal nerve fibre layer thickness (mRNFLt), and retinal nerve fibre cross-sectional area (RNFLcsa), it is necessary to define a reference plane, which is placed at $50 \mu \mathrm{m}$ below the retinal surface within the papillo-macular bundle (the temporal $350-356^{\circ}$ sector), in the software version 1.11. In addition, four other parameters, independent from the reference plane (mean and maximum cup depth, height variation contour, and cup shape measure), were determined.

In order to visualize the three-dimensional shape of the examined surface (quantitatively and qualitatively), a topography image could be displayed. The topography image was enlarged and displayed with a superimposed grid of $16 \times 16$ fields, and each field is $16 \times 16$ picture elements in size. The numeric value indicated an absolute value in micrometres.

\section{Topographic map analysis}

The $40 \mathrm{ONH}$ topographic map data sets were imported and analysed using the MatLab program (The Math Works Inc., Version 5.1).

Analytic-geometric analysis methods were used trying to distinguish ONHs with normal visual field from those with an abnormal one by finding relationship between the observable data and the diagnosis. The details of the topographic map analysis have been described elsewhere. ${ }^{10}$ In brief, three-dimensional images were sectioned into $n$ different contour lines obtained by the intersections of $n$ equidistant planes with the surface $f(x, y)$. Each contour line is constituted of a different number of points of the same height that shape a polygon. The authors used a linear interpolation between the data given by HRT measurements. Using the mentioned criteria, all the images were divided in $n=30$ different contour lines with their relatives areas. The three-dimensional image and its bi-dimensional projection on the $(x, y)$ plane were used to analyse all the ONHs. The authors tuned two complementary methods that could discriminate glaucomatous characteristic from normals.

'Double cup method' (method 1) was defined when there were present two poligonal cups in the polar regions having the same height on the saddle point. An algorithm was used to calculate the cup areas and their ratio. In other words, two depressed areas were present in the superior and inferior areas of the lamina cribrosa as Quigley described. ${ }^{23}$

The second method ('contour line areas') calculated how change the contour line areas among the 30 different planes: in other words the first derivative of the contour line areas was calculated. 


\section{Statistical analysis}

To describe all the considered subjects with standard parameters for each patient disc area, rim volume and cup shape measure were calculated. Besides to compare age, refraction, $\mathrm{ONH}$ parameters, MD and CPSD Student's $t$-test was assessed between the two groups. The sensitivity, the specificity, and the diagnostic precision was calculated for the topographic map analysis program.

\section{Results}

Table 1 lists the details of the recruited patients. No significant difference was found between healthy subjects and glaucoma patients for age, refractive error, and disc area, while significant differences were found for RV, CSM, MD, and CPSD.

When method 1 (double cup method) was applied to the group and in normal ONHs, the value of the ratio between the largest cup and the smallest was more than
10 , while in glaucomatous ONHs, the ratio was $<10$. The presence of 'double cups' was always associated to a pathological condition, even if vice versa was not true. This was a sufficient but not a necessary condition (Figure 1).

Table 1 Descriptive analysis

\begin{tabular}{lccccccc}
\hline & \multicolumn{2}{c}{$\begin{array}{c}\text { Healthy } \\
(\mathrm{n}=20)\end{array}$} & & \multicolumn{2}{c}{$\begin{array}{c}\text { Glaucoma } \\
(\mathrm{n}=20)\end{array}$} & P-value \\
\cline { 2 - 3 } & Mean & $S D$ & & Mean & $S D$ & \\
\hline Age (years) & 63.8 & 4.1 & & 64.3 & 4.7 & NS \\
Refractive error (diopter) & -0.7 & 4.6 & & -1.01 & 5.4 & NS \\
DA (mm $\left.{ }^{2}\right)$ & 2.44 & 0.65 & & 2.49 & 0.64 & NS \\
RV (mm $\left.\mathrm{mm}^{3}\right)$ & 0.41 & 0.20 & & 0.33 & 0.26 & $* * *$ \\
CSM & -0.21. & 0.07 & -0.14 & 0.10 & $* * *$ \\
MD (dB) & -0.43 & 1.33 & -5.15 & 3.85 & $* * *$ \\
CPSD (dB) & 1.45 & 0.98 & 6.03 & 3.58 & $* * *$ \\
\hline
\end{tabular}

NS: not significant, ${ }^{* * *} P<0.001, n$ : number of eyes.

SD: standard deviation, DA: disc area, RV: rim volume, CSM: cup shape measure, MD: mean deviation, CPSD: corrected pattern standard deviation.
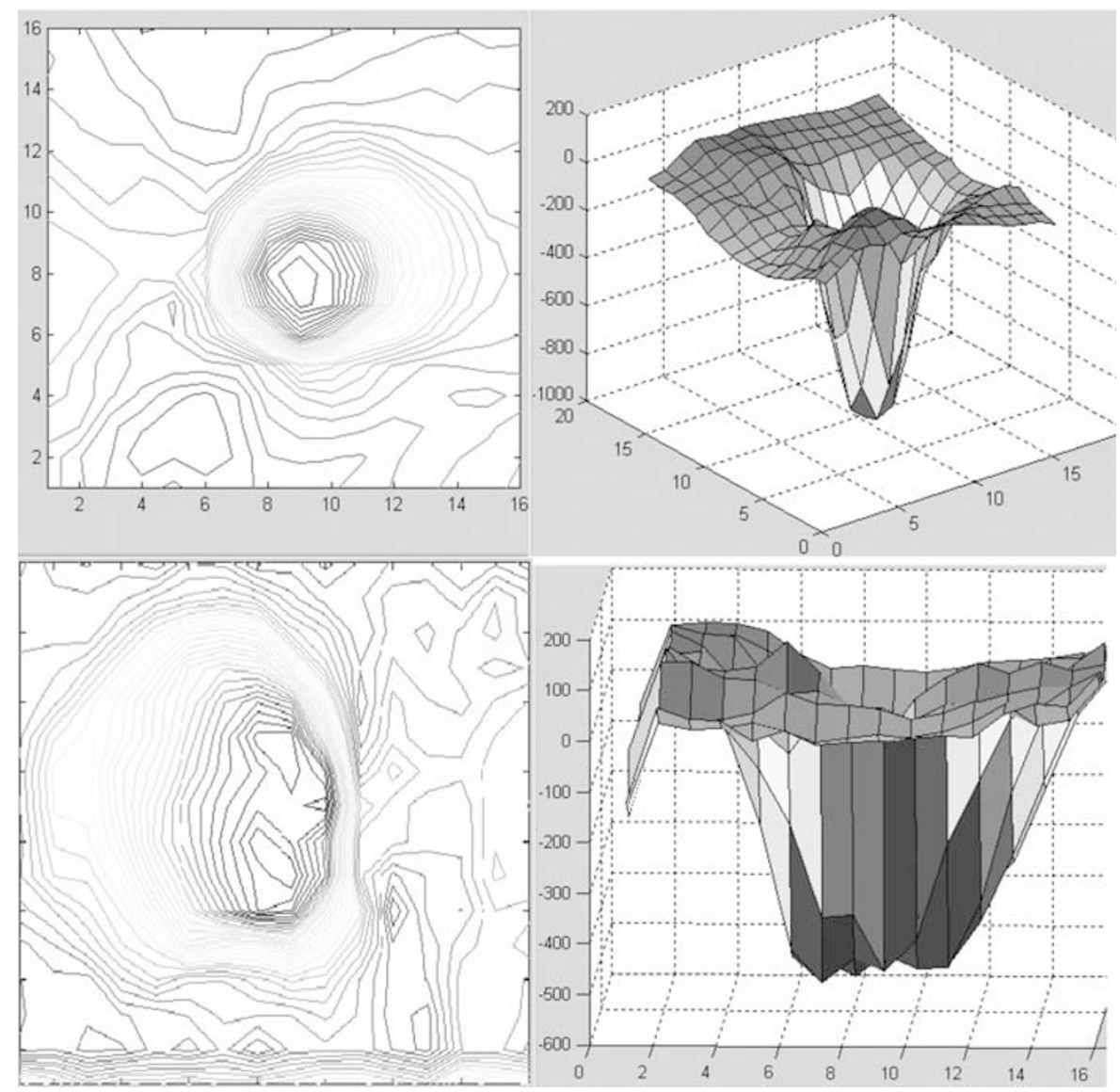

Figure 1 Print out of the results obtained by the topographic map analysis program. Above: Normal ONH map. Right image: Threedimensional image of a normal ONH. Left top image: Bi-dimensional projection on the $(x, y)$ plane of a normal ONH. Each contour line has the same height. Below: Bi-dimensional projection on the $(x, y)$ plane of a glaucomatous ONH. Each contour line has the same height. On the button, this patient has two cups on the superior and inferior pole. 
Table 2 Diagnostic capacity of the topographic map analysis

\begin{tabular}{lccc}
\hline \multicolumn{3}{c}{ New algorithm } \\
\cline { 2 - 3 } & Healthy & Glaucoma & \\
\hline Visual field classification & & $\mathrm{SS}=80 \%$ \\
Healthy & 15 & 5 & $\mathrm{SP}=75 \%$ \\
Glaucoma & 4 & 16 & $\mathrm{DP}=77.5 \%$ \\
\end{tabular}

$\mathrm{SS}=$ sensibility $\mathrm{SP}=$ specificity $; \mathrm{DP}=$ diagnostic capacity.

For the second method, the variation of the contour line areas was considered abnormal when the first derivative of these areas was more than 2 for all the first five consecutive levels or when there was a variation more than 4 between two close areas. When the data of contour line area variation were represented in a graph, the curves were similar as morphology but different in values between normal and glaucomatous optic discs. Indeed, in glaucomatous $\mathrm{ONH}$, the level curve areas changed faster than in normal (Figure 1).

When the topographic map analysis was applied to the group, a sensitivity of $80 \%$, a specificity of $75 \%$, and a diagnostic precision of $77.5 \%$ were obtained (Table 2 ).

\section{Discussion}

It has been widely accepted that analysis of the anatomical changes of the $\mathrm{ONH}$ has a relevant place in establishing the diagnosis of glaucoma. ${ }^{23}$ It has also been shown that in glaucoma structural damage to the $\mathrm{ONH}$ can be recognized prior to the detectable visual field loss. $^{24}$

Analysing all the ONH area, the HRT is able to give a three-dimensional map of the disc. Even if each image is made by many points, users prefer to employ standard parameters for each system and do not use all the available data. ${ }^{13-22}$

To obtain parameter measurements, an outline had to be drawn around the outer optic disc edge and although this technique had a good reproducibility, the contour line position could change from one center to another. ${ }^{10}$

The anatomical variability of normal $\mathrm{ONH}$ shape makes it difficult to differentiate normal from glaucomatous optic discs. ${ }^{25}$ Even a careful clinical $\mathrm{ONH}$ evaluation by visual means is sometimes unable to detect damage in patients who have glaucomatous field loss. Although it is possible that machines will do no better, it seems worthwhile to explore the diagnostic possibilities offered by computerized ONH shape analysis.

In 1997, Rolando et $a l^{26,27}$ introduced a new optic disc morphometric parameter, the optic disc surface smoothness (ODSS), based on the ability of these computerized system to provide the position of a large number of points placed on the surface of the ONH. The ODSS was the results of the evaluation of the relative position of a large number of points located on the surface of the $\mathrm{ONH}$, and it was thought to be less influenced by the reference plane and not by the contourline. This parameter was applied to the Image-net topographic map with a sensitivity and specificity of 88 and $97.4 \%$, respectively. However, when this algorithm was applied to the HRT topographic maps, a worse diagnostic capacity was found (Iester, unpublished data).

Caprioli et al found that mean peripapillary slope of the nerve fibre layer surface can be used to discriminate between healthy subjects and glaucoma patients with clinically useful diagnostic precision (83\%). This parameter was independent of a retinal reference plane and may be particularly useful to detect progressive glaucoma damage. ${ }^{28}$

Using the HRT topographic maps, Iester et al introduced a different way to evaluate ONHs. This new approach was based on the analysis of 65536 points which the HRT measured. Using an 'Euritics Analysis', two different methods were found to be able to differentiate healthy and glaucomatous ONHs. This new approach was based on the possibility to evaluate all the points within the $10^{\circ}$ of the image, and not only using the point within the contourline. The novelty of this method, however, is to avoid the observer input (the $\mathrm{ONH}$ contour line) and to use the topographic map and not only some standard HRT parameters, in order to analyse all the significant $\mathrm{ONH}$ points. ${ }^{10}$

In 2000, also Swindale et $a l^{29}$ introduced a new method to analyse $\mathrm{ONH}$ without using a contorline analysing the shape of the ganglion cells when they cross the scleral canal, and they obtained a good sensitivity and specificity.

The standard HRT analysis (program: Stereometric Measurements) is not entirely automatic, but requires that an operator first draw an outline manually around the edge of the optic nerve. Most of the HRT shape parameters are sensitive to the determination of this outline for the contourline area and for the reference plane position. The placement of the outline is not always obvious; furthermore, the size of the optic discs could be different for many reason: racial, ${ }^{25}$ selection of the subjects, ${ }^{30-32}$ systems used to analyse the ONHs and observers.

From these results, using the HRT topographic map analysis, it was possible to differentiate normal from glaucomatous with a diagnostic capacity similar to discriminate formula analysis, but without any observer input. New ONH shape parameters will be introduced to better use all the topographic map information. 


\section{Acknowledgements}

This study was presented in part at the ARVO (Association for Research in Vision and Ophthalmology) 2004, in Ft Lauderdale, FL, USA. Each author states that he has no proprietary interest in development or marketing of any product or instrument mentioned in this article.

\section{References}

1 Uchida H, Brigatti L, Caprioli J. Detection of structural damage from glaucoma with confocal laser image analysis. Invest Ophthalmol Vis Sci 1996; 37: 2393-2401.

2 Iester M, Mikelberg FS, Swindale NV, Drance SM. ROC analysis of Heidelberg Retina Tomograph optic disc shape measure in glaucoma. Can J Ophthalmol 1997; 32: 382-388.

3 Mikelberg FS, Parfitt CM, Swindale NV, Graham SL, Drance SM, Gosine R et al. Ability of the Heidelberg Retina Tomograph to detect early glaucomatous visual field loss. J Glaucoma 1995; 4: 242-247.

4 Bathija R, Zangwill L, Berry CC, Sample P, Weinreb R. Detection of early glaucomatous structural damage with confocal scanning laser tomography. J Glaucoma 1998; 7: 121-127.

5 Wollstein G, Garway-Heath DF, Hitchings RA. Identification of early glaucoma cases with the scanning laser ophthalmoscope. Ophthalmology 1998; 105: 1557-1563.

6 Bowd C, Chan K, Zangwill LM, Goldbaum MH, Lee TW, Sejnowski TJ et al. Comparing neural networks and linear discriminant functions for glaucoma detection using confocal scanning laser ophthalmoscopy of the optic disc. Invest Ophthalmol Vis Sci 2002; 43: 3444-3454.

7 Iester M, Mardin CY, Budde WM, Junemann AG, Hayler $\mathrm{JK}$, Jonas JB et al. Discriminant analysis formulas of optic nerve head parameters measured by confocal scanning laser tomography. J Glaucoma 2002; 11: 97-104.

8 Ford BA, Artes PH, McCormick TA, Nicolela MT, LeBlanc $\mathrm{RP}$, Chauhan BC. Comparison of data analysis tools for detection of glaucoma with the Heidelberg Retina Tomograph. Ophthalmology 2003; 110: 1145-1150.

9 Iester M, Mikelberg FS, Courtright P, Burk RO, Caprioli J, Jonas JB et al. Interobserver variability of optic disk variables measured by confocal scanning laser tomography. Am J Ophthalmol 2001; 132: 57-62.

10 Iester M, De Ferrari R, Zanini M. Topographic analysis to discriminate glaucomatous from normal optic nerve heads with a confocal scanning laser: new optic disk analysis without any observer input. Surv Ophthalmol 1999; 44: s33-s40.

11 European Glaucoma Society. Terminology and Guidelines for Glaucoma. Savona: Dogma, 1998; pp 64-65.

12 Caprioli J. The contour of the juxtapapillary nerve fiber layer in glaucoma. Ophthalmology 1990; 97: 358-366.

13 Kruse FE, Burk RO, Volcker HE, Zinser G, Harbarth U. Reproducibility of topographic measurements of the optic nerve head with laser tomographic scanning. Ophthalmology 1989; 96: 1320-1324.

14 Dreher AW, Weinreb RN. Accuracy of topographic measurements in a model eye with the laser tomographic scanner. Invest Ophthalmol Vis Sci 1991; 32: 2992-2996.
15 Dreher AW, Tso PC, Weinreb RN. Reproducibility of topographic measurements of the normal and glaucomatous optic nerve head with the laser tomographic scanner. Am J Ophthalmol 1991; 111: 221-229.

16 Cioffi GA, Robin AL, Eastman RD, Perell HF, Sarfarazi FA, Kelman SE et al. Confocal laser scanning ophthalmoscope: reproducibility of optic nerve head topographic measurements with the confocal scanning laser ophthalmoscope. Ophthalmology 1993; 100: 57-62.

17 Lusky M, Bosem ME, Weinreb RN. Reproducibility of optic nerve topography measurements in eyes with undilated pupils. J Glaucoma 1993; 2: 104-109.

18 Mikelberg FS, Wijsman K, Schulzer M. Reproducibility of topographic parameters obtained with the Heidelberg Retina Tomograph. J Glaucoma 1993; 2: 101-103.

19 Rohrschneider K, Burk ROW, Volcker HE. Reproducibility of topographic data acquisition in normal and glaucomatous optic nerve heads with the laser tomographic scanner. Graefes Arch Clin Exp Ophthalmol 1993; 231: 457-464.

20 Weinreb RN, Lusky M, Bartsch DU, Morsman D. Effect of repetitive imaging on topographic measurements of the optic nerve head. Arch Ophthalmol 1993; 111: 636-638.

21 Chauhan BC, LeBlanc RP, McCormick TA, Rogers JB. Test-retest variability of topographic measurements with confocal scanning laser tomography in patients with glaucoma and control subjects. Am J Ophthalmol 1994; 118: 9-15.

22 Zangwill L, Irak I, Berry CC, Garden V, de Souza Lima M, Weinreb RN. Effect of cataract and pupil size on image quality with confocal scanning laser ophthalmoscopy. Arch Ophthalmol 1997; 115: 983-990.

23 Quigley II HA. Changes in the appearance of the optic disc. Surv Ophthalmol 1985; 30: 117-126.

24 Quigley HA, Dunkelberg GR, Green WR. Retinal ganglion cell atrophy correlated with automated perimetry in human eyes with glaucoma. Am J Ophthalmology 1989; 107: 453-456.

25 Tsai CS, Zangwill L, Gonzalez C, Irak I, Garden V, Hoffman $\mathrm{R}$, Weinreb RN et al. Ethnic differences in optic nerve head topography. J Glaucoma 1995; 4: 248-257.

26 Rolando M, Macrí A, Altieri M, Iester M. Morphometric analysis of the optic disc surface. The level of smoothness as a diagnostic parameter for glaucoma. Int Ophthalmol 1997; 20: $15-20$.

27 Rolando M, Macrí A, Iester M. Optic disc surface smoothness and visual field indices. Graefes Arch Clin Exp Ophthalmol 1999; 237: 372-376.

28 Caprioli J, Park HJ, Ugurlu S, Hoffman D. Slope of the peripapillary nerve fiber layer surface in glaucoma. Invest Ophthalmol Vis Sci 1998; 39: 2321-2328.

29 Swindale NV, Stjepanovic G, Chin A, Mikelberg FS. Automated analysis of normal and glaucomatous optic nerve head topography images. Invest Ophthalmol Vis Sci 2000; 41: 1730-1742.

30 Jonas JB, Konigsreuther KA. Optic disk appearance in ocular hypertensive eyes. Am J Ophthalmol 1994; 117: 732-740.

31 Zangwill LM, Van Horn S, De Souza Lima M, Sample PA, Weinreb RN. Optic nerve head topography in ocular hypertensive eyes using confocal scanning laser ophthalmoscopy. Am J Ophthalmol 1996; 122: 520-525.

32 Iester M, Broadway DC, Mikelberg FS, Drance SM. A comparison of normal, ocular hypertension and glaucomatous optic disc topographic parameters. J Glaucoma 1997; 6: 363-370. 